\title{
SOLAR RADIANT HEATING OF A ROTATING SOLID CYLINDER*
}

By D. A. PRELEWICZ AND LAWRENCE A. KENNEDY (State University of New York at Buffalo)

In an earlier paper, Olmstead and Raynor (1) have considered the temperature distribution in a solid cylinder exposed to solar radiation. They obtained a solution using the method of Green's functions and numerical examples were presented utilizing asymptotic expansions of the modified Bessel functions appearing in the formal solution.

In the present note, a Fourier series expansion of the radiation input function is used in conjunction with a simple product solution to obtain the results in a straightforward manner.

Using the notation of [1], the governing equation, in a coordinate system fixed relative to the incident radiation is

$$
\frac{\partial^{2} \tilde{T}}{\partial s^{2}}+\frac{1}{s} \frac{\partial \tilde{T}}{\partial s}+\frac{1}{s^{2}} \frac{\partial^{2} \tilde{T}}{\partial \theta^{2}}+\zeta_{0} \frac{\partial \tilde{T}}{\partial \theta}=0
$$

with the boundary condition on the outside surface.

$$
\frac{\partial \tilde{T}}{\partial s}+\beta \tilde{T}=-\frac{\beta}{4}+\frac{\pi}{4} \beta \cos \theta H(\cos \theta) .
$$

Here $\tilde{T}$ is the dimensionless temperature fluctuation about an average temperature $T_{0}$, $\boldsymbol{s}$ is the dimensionless radius and $\zeta_{0}$ is the dimensionless angular velocity. $\beta$ is defined as a dimensionless parameter $\beta=\left(4 b \sigma e T_{0}{ }^{3} / k\right)$ and $H(\cos \theta)$ is the Heaviside step function defined by

$$
\begin{aligned}
H(\cos \theta) & =1, \text { for } 0 \leq \theta \leq \pi, \\
& =0, \text { for } \pi \leq \theta \leq 2 \pi .
\end{aligned}
$$

First expand the solar radiation input, $\cos \theta H(\cos \theta)$, in a Fourier series.

$$
\cos \theta H(\cos \theta)=\sum_{n=1}^{\infty}\left[A_{n} \cos n \theta+B_{n} \sin n \theta\right]+\frac{1}{2} A_{0} .
$$

After evaluating the coefficients this becomes

$$
\cos \theta H(\cos \theta)=\frac{1}{\pi}+\frac{1}{2} \cos \theta+\sum_{n=2}^{\infty} \frac{2(-1)^{1+n / 2}}{\pi\left(n^{2}-1\right)} \cos n \theta .
$$

Solution. Since the temperature distribution must be periodic, consider a solution of the form

$$
\tilde{T}=C e^{-i n \theta} P(s)
$$

where continuity requires that $n$ be an integer.

Substituting into Eq. (1) yields

$$
\frac{d^{2} P}{d s^{2}}+\frac{1}{s} \frac{d P}{d s}-\left(i \zeta_{0} n+\frac{n^{2}}{s^{2}}\right) P=0
$$

*Received July 14, 1966; revised manuscript received October 7, 1966. 
which is a form of Bessel's equation having solutions

$$
P(s)=C_{0}, \quad P(s)=C_{n} I_{n}\left(\left(i n \zeta_{0}\right)^{1 / 2} s\right) .
$$

Here the condition of regularity at $s=0$ has been used and $I_{n}$ is the modified Bessel function.

Let $\lambda_{n} \equiv\left(i n \zeta_{0}\right)^{1 / 2}$. Then our temperature distribution $\tilde{T}$ is given by

$$
\tilde{T}=C_{0}+\sum_{n=1}^{\infty}\left[\left\{C_{n} I_{n}\left(\lambda_{n} s\right)+D_{n} I_{n}\left(i \lambda_{n} s\right)\right\} \cos n \theta+i\left\{D_{n} I_{n}\left(2 \lambda_{n} s\right)-C_{n} I_{n}\left(\lambda_{n} s\right)\right\} \sin n \theta\right] .
$$

Applying the boundary conditions at the surface and equating coefficients gives

$$
C_{n}=\frac{Z_{n}}{2 X_{n}}, \quad D_{n}=\frac{Z_{n}}{2 Y_{n}}, \quad C_{0}=0
$$

swhere

$$
\begin{array}{rlrl}
X_{n} & =\lambda_{n} I_{n}^{\prime}\left(\lambda_{n}\right)+\beta I_{n}\left(\lambda_{n}\right), \\
Y_{n} & =i \lambda_{n} I_{n}^{\prime}\left(i \lambda_{n}\right)+\beta I_{n}\left(i \lambda_{n}\right), \\
Z_{n} & =\frac{1}{8} \pi \beta, \quad n=1, \\
& =\frac{\beta(-1)^{1+n / 2}}{2\left(n^{2}-1\right)}, & n=2,4,6 \cdots, \\
& =0, & n=3,5,7, \cdots .
\end{array}
$$

Thus

$$
\tilde{T}^{\prime}=\sum_{n=1}^{\infty}\left[\frac{Z_{n} I_{n}\left(\lambda_{n} s\right) e^{-i n \theta}}{2\left\{\lambda_{n} I_{n}^{\prime}\left(\lambda_{n}\right)+\beta I_{n}\left(\lambda_{n}\right)\right\}}+\frac{Z_{n} I_{n}\left(i \lambda_{n} s\right) e^{i n \theta}}{2\left\{i \lambda_{n} I_{n}^{\prime}\left(i \lambda_{n}\right)+\beta I_{n}\left(i \lambda_{n}\right)\right\}}\right]
$$

Since the coefficients in the summation are the complex conjugate of each other this may be written

$$
\tilde{T}^{\prime}=\sum_{n=1}^{\infty}\left\{Z_{n} \operatorname{Re}\left[\frac{I_{n}\left(\lambda_{n} s\right)}{\lambda_{n} I_{n}^{\prime}\left(\lambda_{n}\right)+\beta I_{n}\left(\lambda_{n}\right)}\right] \cos n \theta+Z_{n} \operatorname{Im}\left[\frac{I_{n}\left(\lambda_{n} s\right)}{\lambda_{n} I_{n}^{\prime}\left(\lambda_{n}\right)+\beta I_{n}\left(\lambda_{n}\right)}\right] \sin n \theta\right\} .
$$

Then using $T=T_{0}(1+\tilde{T})$, the total temperature can be obtained. This yields

$$
\frac{T}{T_{0}}=1+\beta\left\{\frac{\pi}{8}\left[a_{1} \cos \theta+b_{1} \sin \theta\right]+\frac{1}{2} \sum_{n=1}^{\infty} \frac{(-1)^{n+1}}{\left(4 n^{2}-1\right)}\left[a_{2 n} \cos 2 n \theta+b_{2 n} \sin 2 n \theta\right]\right\}
$$

where

$$
\begin{aligned}
& a_{n} \equiv \operatorname{Re}\left\{\frac{I_{n}\left(\lambda_{n} s\right)}{\lambda_{n} I_{n}^{\prime}\left(\lambda_{n}\right)+\beta I_{n}\left(\lambda_{n}\right)}\right\}, \\
& b_{n} \equiv \operatorname{Im}\left\{\frac{I_{n}\left(\lambda_{n} s\right)}{\lambda_{n} I_{n}^{\prime}\left(\lambda_{n}\right)+\beta I_{n}\left(\lambda_{n}\right)}\right\}
\end{aligned}
$$

This solution is identical to that obtained by Olmstead and Raynor [1] through the more laborious and elegant Green function approach. Rather than expressing this 
result in terms of $b e r_{n}$ and $b e i_{n}$ functions and performing asymptotic expansions, numerical examples can be obtained utilizing a digital computer program for calculating the modified Bessel functions.

\section{REFERENCE}

[1] W. E. Olmstead and S. Raynor, Solar heating of a rotating solid cylinder, Quart. Appl. Math. 21, 81 (1963) 